\title{
Original
}

\section{Growth of an aggressive tumor during pregnancy in an acromegalic patient}

\author{
Leandro Kasuki ${ }^{1), 3)}$, Leonardo Vieira Neto ${ }^{1)}{ }^{4)}$, Christina M. Takiya ${ }^{5)}$ and Mônica R. Gadelha ${ }^{1), 2)}$ \\ ${ }^{1)}$ Serviço de Endocrinologia, Hospital Universitário Clementino Fraga Filho, Rio de Janeiro, Brazil \\ ${ }^{2)}$ Instituto Estadual de Diabetes e Endocrinologia Luiz Capriglione, Rio de Janeiro, Brazil \\ ${ }^{3)}$ Serviço de Endocrinologia, Hospital Federal de Bonsucesso, Brazil \\ ${ }^{4)}$ Serviço de Endocrinologia, Hospital Federal da Lagoa, Brazil \\ ${ }^{5)}$ Laboratorio de Patologia Celular, UFRJ, Rio de Janeiro, Brazil
}

\begin{abstract}
Pregnancy in acromegalic patients is a rare event, but is usually uneventful, with stable GH and insulin-like growth factor I (IGF-I) levels and no tumor enlargement. Medical treatment can usually be withdrawn without problems and although no major adverse event has been reported, the suspension of drug treatments is generally recommended. No case report exists in the literature regarding evolution of a somatotropinoma with invasiveness markers throughout pregnancy. We report a case of an acromegalic patient who was submitted to surgery and treated with octreotide LAR maintaining a stable residual tumor and an IGF-I close to the normal levels. Her tumor presented with a high Ki-67 (11.6\%) and a low aryl hydrocarbon receptor-interacting protein (AIP) expression. When she became pregnant, octreotide LAR was withdrawn, and despite remaining asymptomatic during pregnancy, tumor growth occurred with compression of surrounding structures. In conclusion, pregnancy in acromegalic patients has usually a favorable prognosis with no tumor growth. However, in the presence of high Ki-67 labeling index and low AIP expression, tumor enlargement may occur and somatostatin analogue treatment throughout the pregnancy should be considered.
\end{abstract}

Key words: Acromegaly, Pregnancy, Aryl hydrocarbon receptor-interacting protein, Ki-67, Aggressive adenomas

FERTILITY is usually compromised in acromegaly due to hyperandrogenemia, hyperprolactinemia and impairment of gonadotropin-secreting cells as a result of tumor mass effect or treatment (surgery and radiotherapy) [1, 2]. Thus, pregnancy is a rare event in acromegalic patients, and few cases have been described [3-5]. However, with the improvement in treatment modalities, pregnancy in acromegaly will become more frequent.

The behavior of GH and insulin-like growth factor I (IGF-I) levels is controversial during pregnancy, but tumor growth is not observed in the majority of the cases [2, 4-6]. No information is available in the literature regarding the outcome of pregnancy in acromegalic patients with invasiveness markers.

Submitted Oct. 21, 2011; Accepted Jan. 1, 2012 as EJ11-0306 Released online in J-STAGE as advance publication Jan. 12, 2012

Correspondence to: Mônica R. Gadelha, M.D., Ph.D., Centro de Pesquisa em Neuroendocrinologia, Rua Professor Rodolpho Paulo Rocco, 255, sala 9F, Ilha do Fundão, Rio de Janeiro 21941-913, Brazil. E-mail: mgadelha@hucff.ufrj.br
In this article, we describe a case of an acromegalic woman whose tumor presented with high Ki-67 and low aryl hydrocarbon receptor interacting-protein (AIP) expression, in whom tumor growth was observed during pregnancy.

\section{Subjects and Methods}

The patient is currently being followed in the endocrinology unit of the Hospital Universitário Clementino Fraga Filho/Universidade Federal do Rio de Janeiro and signed informed consent was obtained.

\section{Immunohistochemistry}

Paraffin-embedded tissue sections $(4 \mu \mathrm{m})$ were dewaxed and, after rehydration, were submitted to heat-mediated antigen retrieval for Ki-67 in Dako ${ }^{\circledR}$ Retriever buffer (Dako, Carpinteria, CA, USA), pH 9.0 in a steamer for 30 minutes, for AIP in Tris-EDTA buffer, pH 9.0 in a microwave for 5 minutes at $750 \mathrm{~W}$ [7] and for somatostatin receptor subtype 2 (SSTR2) in citric 
acid buffer $0.01 \mathrm{M}$, pH 6.0 in microwave for 10 minutes at $750 \mathrm{~W}$ [8]. Endogenous peroxidase was inhibited in $0.3 \%$ hydrogen peroxide for 10 minutes, followed by blocking unspecific immunoglobulin binding with $5 \%$ bovine serum albumin in phosphate saline buffer $(\mathrm{pH}$ 7.4), before primary antibody incubation.

Mouse monoclonal antibodies directed against Ki-67 antigen (1:100, Dako, MIB-1 clone, cat. number M-7240) and AIP (1:500, Novus Biological, Littleton, CO, USA, 35-2 clone, cat. number NB100-127) were used. Policlonal antibodies anti-SSTR2A (SS-800, Gramsch Laboratories, Schwabhausen, Germany) were used. Antibodies were revealed with the EnVision peroxidase kit (Dako, cat. number K1491) and peroxidaselabeled, polymer-conjugated secondary antibodies, followed by the chromogen substrate diaminobenzidine (Liquid DAB, Dako, cat. number K3468). Tissues were then lightly counterstained with hematoxylin.

For Ki-67, human breast carcinoma was used as positive control, whereas normal human pituitary was used for AIP and SSTR2. Negative controls were performed by incubating in the isotype immunoglobulin or omitting the primary antibody.

Histomorphometry was performed using ImagePro Plus 4.5.1 (Media Cybernetics, Silver Spring, MD, USA) coupled to a digital camera (Evolution, Media Cybernetics) and a light microscope (Eclipse 400, NIKON, Japan). For Ki-67, high-quality images (2048 $\mathrm{x} 1536$ pixels) of immunostained tumor cells from 10 representative areas with at least 1000 cells were captured using a 40x objective lens. Both immunolabeled and unlabeled nuclei were evaluated, and the percentage of positive cells (labeling index - LI) was calculated. For AIP, high-quality images (2048 x 1536 pixels) of immunostained tumor cells from 20 random areas were captured using a 20x objective lens. For semi-quantitative estimate of cytoplasmic AIP immunostaining, slides were scored for pattern (diffuse (score 2) or patchy (score 1)) and for intensity (strong (score 3), moderate (score 2) and weak (score 1)), and the final score was calculated by multiplying the two (pattern and intensity) scores, as previously described [7, 9]. Scores 0 (no expression), 1 and 2 are considered low AIP expression [7]. For SSTR2, high quality images (2048 X 1536 pixels buffer) of immunostained tumor cells were randomly captured using a $40 \mathrm{x}$ objective lens (10 microscopical fields). Tumor was staged according to the percentage of stained cells in score: 0 ( $<25 \%$ stained cells - low expression), 1 (25-50\% - moderate expression) or 2 ( $>50 \%$ - high expression). Both membrane-bound and intracytoplasmic immunopositivity were considered for staging [8].

\section{Analysis of AIP mutation}

Genomic deoxyribonucleic acid (DNA) was extracted using QIAamp DNA MiniKit (Qiagen, Valencia, CA) from peripheral white blood cells, according to manufacturer's protocol. The entire coding sequence of AIP (NM_003977.2), conserved splice sites (from the conserved A of the upstream branch site to +10 downstream of each exon) and 1200 base-pairs of the promoter region were direct sequenced. The MLPA (P244-B1 kit MRC-Holland, Amsterdam, Netherlands) dosage analysis was carried out to look for partial or whole gene deletions.

\section{Case Report}

A 26-year-old female patient presented with headache, paresthesias and enlargement of hands and feet, and the diagnosis of acromegaly was made: basal GH was $16.2 \mathrm{ng} / \mathrm{mL}$ and IGF-I was $734 \mathrm{ng} / \mathrm{mL}$ (normal range (NR): 117-321); the prolactin level was slightly elevated: $47 \mathrm{ng} / \mathrm{mL}$ (NR: 9-23). The rest of pituitary function was normal. Pituitary magnetic resonance imaging (MRI) showed a macroadenoma of $2.2 \times 1.7$ x 1.5 $\mathrm{cm}$ with suprasellar and right cavernous sinus extension (Fig. 1). The patient underwent adenomectomy by the transsphenoidal approach, with significant tumor removal (tumor size after surgery: $0.9 \times 1.3 \times 1.5 \mathrm{~cm}$ ) and reduction of GH (basal $4.7 \mathrm{ng} / \mathrm{mL}$, nadir on OGTT 3.3 $\mathrm{ng} / \mathrm{mL})$ and IGF-I (528 ng/mL) levels. Her pituitary function at this time was normal, including the prolactin levels $(7.3 \mathrm{ng} / \mathrm{mL})$. The tumor presented with high cellularity and nuclear pleomorphism (approximately $10 \%$ of the nuclei), with some mitoses and apoptotic cells. There was positive immunostaining for GH and negative staining for all other pituitary hormones, including prolactin. The SSTR2 was expressed in 90\% of the cells. The tumor also had a KI-67 LI of $11.6 \%$ and a low AIP expression (score 1 - patchy and weak) (Fig. 2). She had no familial history of pituitary adenomas, but as she was a young patient, screening for AIP mutation was done, but it was negative.

Octreotide LAR was started after surgery at an initial dose of $20 \mathrm{mg}$ every 28 days. After 6 months of octreotide LAR in this dosage, tumor size was stable and she maintained a GH of $7.5 \mathrm{ng} / \mathrm{mL}$ and an IGF-I 


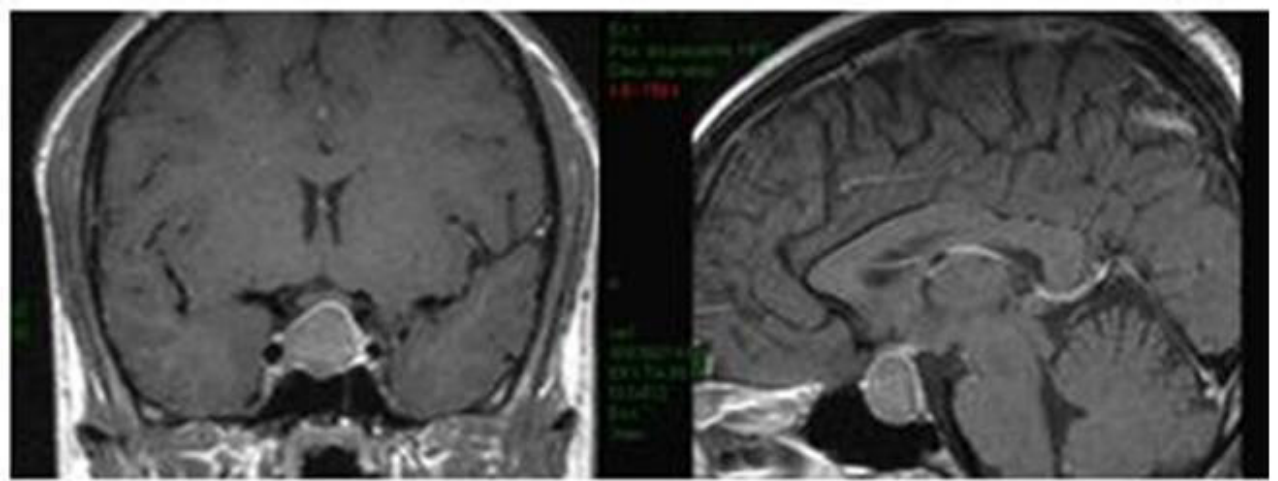

Fig. 1 Pituitary magnetic resonance imaging showing a macroadenoma of 2.2 x 1.7 x $1.5 \mathrm{~cm}$ with right cavernous sinus and suprasellar extension (abutting the optic chiasm) 254x190mm (72 x 72 DPI)
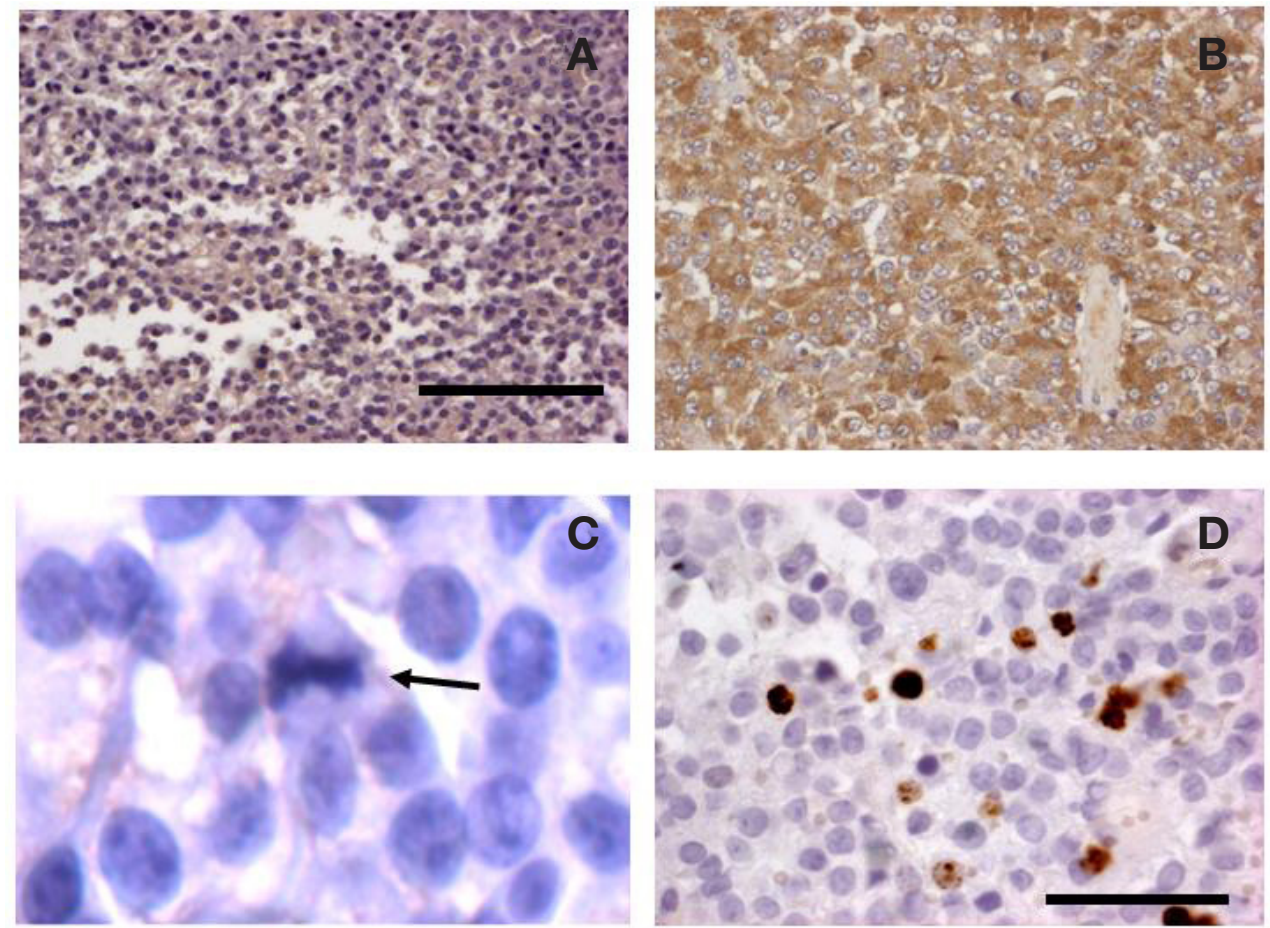

Fig. 2 AIP immunostaining (A and B). A - Patient's AIP: score 1 (weak and patchy). Scale bar $=100 \mu$ m. B - Example of tumor with AIP score 6 (strong and diffuse). C - Mitose in the tumor (arrow). D - Patient's positive nuclear Ki-67 immunostaining. Scale bar $=500 \mu \mathrm{m} .254 \times 190 \mathrm{~mm}(72 \times 72$ DPI $)$

of $341 \mathrm{ng} / \mathrm{mL}$, so the dosage was increased to $30 \mathrm{mg}$ every four weeks. However, she became pregnant after the first injection of octreotide LAR $30 \mathrm{mg}$ and the drug was discontinued.

During pregnancy, the patient remained clinically asymptomatic and maintained a high GH and an IGF-I close to the upper limit of normality (ULN) (Table 1). Pregnancy period was uneventful and the patient remained without arterial hypertension or glu- cose abnormalities. Cesarean delivery took place at 37 weeks as an obstetrics choice due to lack of dilatation of the cervix on labor. The newborn was healthy with normal weight and length. Two weeks after delivery, the patient complained of headache, hands and feet edema, severe asthenia and hyperidrosis. Laboratory evaluation showed a GH of $18.1 \mathrm{ng} / \mathrm{mL}$ and an increase in IGF-I from $377 \mathrm{ng} / \mathrm{mL}$ (one month earlier, at 34 weeks of pregnancy) to $1076 \mathrm{ng} / \mathrm{mL}$. In the pre-gesta- 
Table 1 Evolution of GH and IGF-I levels

\begin{tabular}{lcc}
\hline & GH & IGF-I (NR:117-321) \\
\hline Diagnosis & 16.2 & 734 \\
Post surgery & 4.7 & 528 \\
Octreotide LAR 20mg 6 months (pre-gestational) & 7.5 & 341 \\
Pregnancy first trimester & 9.1 & 462 \\
Pregnancy second trimester & 12.3 & 360 \\
Pregnancy third trimester (34 weeks) & 13.8 & 377 \\
Two weeks post-partum & 18.1 & 1076 \\
Octreotide LAR 30 mg 3 months (post-partum) & 18.0 & 533 \\
\hline
\end{tabular}

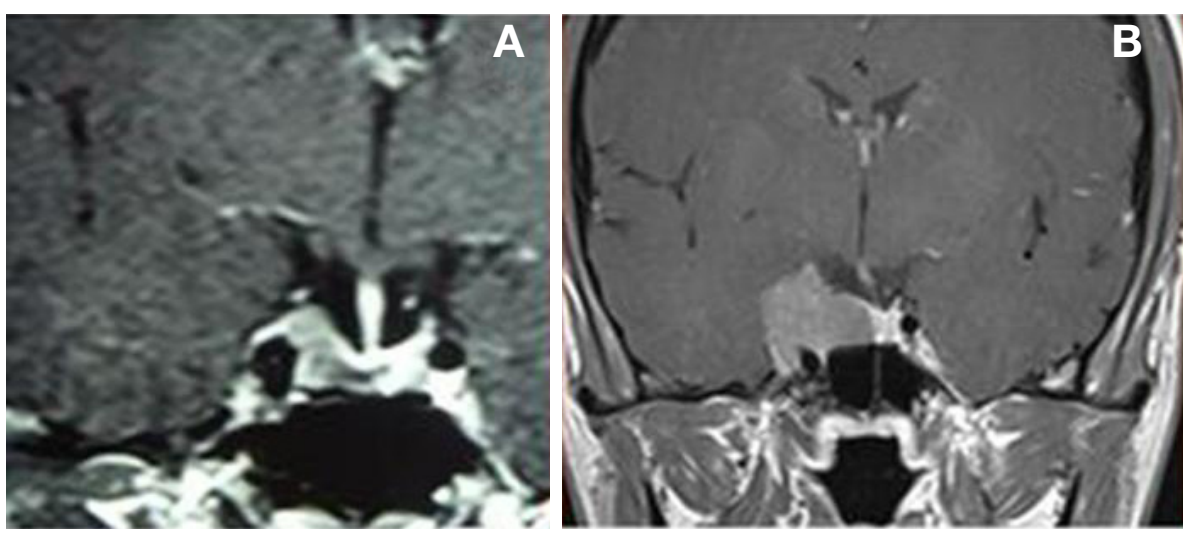

Fig. 3 Pituitary magnetic resonance imaging showing growth of the pituitary tumor from the pregestational (A) to the post-partum (B) moment, invading the cavernous sinus and compressing the temporal lobe 254x190mm (72 x 72 DPI)

tional MRI the tumor measured $0.9 \times 1.3 \times 1.5 \mathrm{~cm}$ while a new MRI at this moment showed an important increase in tumor size $(2.2 \times 1.7 \times 1.7 \mathrm{~cm})$ with invasion of right cavernous sinus and compression of the temporal lobe (Fig. 3). Lactation was discontinued and octreotide LAR at a dosage of $30 \mathrm{mg}$ was restarted. She presented with a decrease in IGF-I, without normalization, maintaining a high GH (Table 1) and no tumor reduction. A second surgery was tried with the same skilled neurosurgeon, however, there was significant bleeding and the cavernous artery was blocking the access to the tumoral tissue, so she was submitted to radiotherapy (Intensity modulated radiotherapy - IMRT) with 50.4 Gy divided in 28 sessions.

\section{Discussion}

Limited data is available about pregnancy in acromegaly $[4,10]$. In relation to hormonal hypersecretion, it is known that in normal pregnancy occurs secretion of placental GH variant, whose levels increase progres- sively replacing pituitary GH around $15-20$ weeks [11, 12]. This elevation in placental GH levels increases IGF-I [11]. However, different from normal pituitary, the placental GH does not inhibit the secretion of GH by somatotropinomas, and therefore it is expected to find increased levels of GH and IGF-I in acromegalic patients. Although earlier data confirm this hypothesis [2], several reports have recently shown improvement of IGF-I levels during pregnancy, which usually are within normal range or around the ULN $[3,5,13]$. We observed the same behavior of the IGF-I levels in our patient, even after cessation of octreotide LAR treatment. In fact, her IGF-I levels remained close to normal, and she did not present with clinical symptoms of active disease. The possible explanation for this is an inhibition of hepatic synthesis of IGF-I by increased estrogen, what is supported by the discordant increase of GH levels in our patient. It is not possible to determine whether this increase was due to tumoral GH, as our assay is not able to distinguish pituitary and placental GH variants. 
Regarding tumor growth during pregnancy, although adenoma enlargement may theoretically be expected in acromegalic patients because of estrogen exposure [2], in the majority of patients tumor enlargement is not observed $[3-5,13,14]$. Some cases of tumor growth have been described, mainly in patients with large tumors not submitted to surgery before pregnancy, which stopped somatostatin analogue treatment [13, 15]. So far, no information regarding the behavior of somatotropinomas with positive invasiveness markers is available in the literature. Despite our patient has been operated on and remained with a relatively small remnant tumor before conception, she presented with a rapid tumor growth during pregnancy, with compression of parasellar structures. In the tumor evaluation we found a high Ki-67 LI and a low AIP expression. Ki-67 is a proliferative marker associated in some studies with tumor invasiveness or recurrence $[16,17]$. Most of these studies adopted a 3\% cut-off point to define a tumor as invasive $[17,18]$. In our previous series, median Ki-67 LI was also higher among invasive somatotropinomas [7]. In this same publication we demonstrated that low AIP expression was associated with invasiveness in somatotropinomas as well, with a better accuracy than Ki-67 LI for this classification. Thus, the tumor growth observed in our patient is probably not related to the pregnancy itself, but to the aggressiveness of the tumor. This hypothesis is corroborated by the literature data, including a recent review [4] that did not observed tumor growth related to pregnancy in acromegaly.

Mutations in the AIP gene are found in about 50\% of familial isolated somatotropinomas and are associated with a more aggressive phenotype [19]. In seemingly sporadic pituitary adenomas, the occurrence of mutations is much less frequent [20, 21]. However, it has been previously described that younger patients with somatotropinomas have a higher frequency of germline mutations, especially those less than 30 years old [22]. As our patient was young and presented with an aggressive tumor, we performed a search for an AIP mutation, but it was negative. Although mutations are rare in sporadic somatotropinomas [20, 21], a subset of invasive tumors present with low AIP protein expression in the absence of mutations, as demonstrated by our group and others $[7,23]$. These data indicate that another mechanism different from mutation is responsible for this low expression. Further studies are necessary to clarify this topic.
In our patient, the withdrawal of octreotide LAR treatment probably contributed to tumor enlargement. Although in most of the previously described cases the patients were not on octreotide LAR therapy or it was stopped when pregnancy was diagnosed, there are some reports in which somatostatin analogs (SA) were maintained throughout the pregnancy without significant side effects on the mother or fetus [13, 24, 25]. A recent publication of two referral centers, with 13 new cases and literature review, suggested that pregnancy in treated acromegalic women can proceed without significant complications or teratogenicity [5]. However, medical treatment with SA during pregnancy was associated with low birth weight. In another recent publication, the effects of octreotide on uterine artery blood flow, octreotide concentrations in biological fluids of mother and newborn, SSTR expression and binding at the level of the maternal-fetal barrier tissues were studied [26]. Only an acute decrease in uterine artery blood flow was observed after octreotide injections, without affecting the pregnancy course, delivery or fetal development. Octreotide concentrations were higher in the maternal serum and colostrum and lower in the umbilical cord, amniotic fluid and newborn serum. In addition, SSTR subtypes were expressed in the placental tissue but their binding profile was weak both in the placenta and umbilical cord. So, despite the concern regarding fetal growth, the treatment of acromegalic patients with octreotide during pregnancy is probably safe for the mother and the fetus and, although not generally recommended, if it had been maintained in our patient, the course could have been different.

In conclusion, pregnancy in acromegalic patients has usually a favorable prognosis, with maintenance of IGF-I levels near the normal range and no tumor growth. However, in the non-cured acromegalic patient after surgery with high Ki-67 LI and low AIP expression, carefully follow-up with regular visual fields should be done. In the presence of worsening of symptoms or visual field abnormalities, a plain pituitary MRI should be done to check if there is tumor enlargement and somatostatin analogue treatment throughout pregnancy should be considered if it is found.

\section{Conflict of Interest Statement}

The authors declare that they have no conflict of interest. 


\section{References}

1. Grynberg M, Salenave S, Young J, Chanson P (2010) Female gonadal function before and after treatment of acromegaly. J Clin Endocrinol Metab 95:4518-4525.

2. Herman-Bonert V, Seliverstov M, Melmed S (1998) Pregnancy in acromegaly: successful therapeutic outcome. J Clin Endocrinol Metab 83:727-731.

3. Cozzi R, Attanasio R, Barausse M (2006) Pregnancy in acromegaly: a one-center experience. Eur J Endocrinol 155:279-284.

4. Cheng V, Faiman C, Kennedy L, Khoury F, Hatipoglu B, Weil R, Hamrahian A (2011) Pregnancy and acromegaly: a review. Pituitary July 26. Epub ahead of print.

5. Cheng S, Grasso L, Martinez-Orozco JA, Al-Agha R, Pivonello R, Colao A, Ezzat S (2011) Pregnancy in Acromegaly: Experience from Two Referral Centers and Systematic Review of the Literature. Clin Endocrinol (Oxf) July 20. Epub ahead of print.

6. Shimatsu A, Usui T, Tagami T, Kuzuya H, Takahashi JA (2010) Suppressed levels of growth hormone and insulin-like growth factor-1 during successful pregnancy in persistent acromegaly. Endocr J 57:551-553.

7. Kasuki Jomori de Pinho L, Vieira Neto L, Armondi Wildemberg LE, Gasparetto EL, Marcondes J, de Almeida Nunes B, Takiya CM, Gadelha MR (2011) Low aryl hydrocarbon receptor-interacting protein expression is a better marker of invasiveness in Somatotropinomas than Ki-67 and p53. Neuroendocrinology 94:39-48.

8. Wildemberg LE, Vieira Neto L, Costa DF, Nasciutti LE, Takiya CM, Alves LM, Gadelha MR (2011) Validation of immunohistochemistry for somatostatin receptor subtype 2A in human somatotropinomas: comparison between quantitative real time RT-PCR and immunohistochemistry. J Endocrinol Invest Sep 6. Epub ahead of print.

9. Leontiou CA, Gueorguiev M, van der Spuy J, Quinton R, Lolli F, Hassan S, Chahal HS, Igreja SC, Jordan S, Rowe J, Stolbrink M, Christian HC, Wray J, BishopBailey D, Berney DM, Wass JA, Popovic V, RibeiroOliveira A, Jr., Gadelha MR, Monson JP, Akker SA, Davis JR, Clayton RN, Yoshimoto K, Iwata T, Matsuno A, Eguchi K, Musat M, Flanagan D, Peters G, Bolger GB, Chapple JP, Frohman LA, Grossman AB, Korbonits M (2008) The role of the aryl hydrocarbon receptor-interacting protein gene in familial and sporadic pituitary adenomas. J Clin Endocrinol Metab 93:2390-2401.

10. Bronstein MD, Paraiba DB, Jallad RS (2011) Management of pituitary tumors in pregnancy. Nat Rev Endocrinol 7:301-310.

11. Beckers A, Stevenaert A, Foidart JM, Hennen G, Frankenne F (1990) Placental and pituitary growth hormone secretion during pregnancy in acromegalic women. J Clin Endocrinol Metab 71:725-731.

12. Frankenne F, Closset J, Gomez F, Scippo ML, Smal J, Hennen G (1988) The physiology of growth hormones (GHs) in pregnant women and partial characterization of the placental GH variant. J Clin Endocrinol Metab 66:1171-1180.

13. Caron P, Broussaud S, Bertherat J, Borson-Chazot F, Brue T, Cortet-Rudelli C, Chanson P (2010) Acromegaly and pregnancy: a retrospective multicenter study of 59 pregnancies in 46 women. J Clin Endocrinol Metab 95:4680-4687.

14. Caron P (2011) Acromegaly and pregnancy. Ann Endocrinol (Paris) 72:282-286.

15. Atmaca A, Dagdelen S, Erbas T (2006) Follow-up of pregnancy in acromegalic women: different presentations and outcomes. Exp Clin Endocrinol Diabetes 114:135-139.

16. Fusco A, Zatelli MC, Bianchi A, Cimino V, Tilaro L, Veltri F, Angelini F, Lauriola L, Vellone V, Doglietto F, Ambrosio MR, Maira G, Giustina A, degli Uberti EC, Pontecorvi A, De Marinis L (2008) Prognostic significance of the Ki-67 labeling index in growth hormonesecreting pituitary adenomas. J Clin Endocrinol Metab 93:2746-2750.

17. OgawaY,IkedaH,TominagaT(2009)Clinicopathological study of prognostic factors in patients with pituitary adenomas and Ki-67 labeling index of more than 3\%. $J$ Endocrinol Invest 32:581-584.

18. Salehi F, Agur A, Scheithauer BW, Kovacs K, Lloyd RV, Cusimano M (2009) Ki-67 in pituitary neoplasms: a review--part I. Neurosurgery 65:429-437.

19. Igreja S, Chahal HS, King P, Bolger GB, Srirangalingam U, Guasti L, Chapple JP, Trivellin G, Gueorguiev M, Guegan K, Stals K, Khoo B, Kumar AV, Ellard S, Grossman AB, Korbonits M (2010) Characterization of aryl hydrocarbon receptor interacting protein (AIP) mutations in familial isolated pituitary adenoma families. Hum Mutat 31:950-960.

20. Barlier A, Vanbellinghen JF, Daly AF, Silvy M, JaffrainRea ML, Trouillas J, Tamagno G, Cazabat L, Bours V, Brue T, Enjalbert A, Beckers A (2007) Mutations in the aryl hydrocarbon receptor interacting protein gene are not highly prevalent among subjects with sporadic pituitary adenomas. J Clin Endocrinol Metab 92:19521955.

21. Raitila A, Georgitsi M, Karhu A, Tuppurainen K, Makinen MJ, Birkenkamp-Demtroder K, Salmenkivi K, Orntoft TF, Arola J, Launonen V, Vahteristo P, Aaltonen LA (2007) No evidence of somatic aryl hydrocarbon receptor interacting protein mutations in sporadic endocrine neoplasia. Endocr Relat Cancer 14:901-906.

22. Cazabat L, Libe R, Perlemoine K, Rene-Corail F, 
Burnichon N, Gimenez-Roqueplo AP, DupasquierFediaevsky L, Bertagna X, Clauser E, Chanson P, Bertherat J, Raffin-Sanson ML (2007) Germline inactivating mutations of the aryl hydrocarbon receptor-interacting protein gene in a large cohort of sporadic acromegaly: mutations are found in a subset of young patients with macroadenomas. Eur J Endocrinol 157:1-8.

23. Jaffrain-Rea ML, Angelini M, Gargano D, Tichomirowa MA, Daly AF, Vanbellinghen JF, D’Innocenzo E, Barlier A, Giangaspero F, Esposito V, Ventura L, Arcella A, Theodoropoulou M, Naves LA, Fajardo C, Zacharieva S, Rohmer V, Brue T, Gulino A, Cantore G, Alesse E, Beckers A (2009) Expression of aryl hydrocarbon receptor (AHR) and AHR-interacting protein in pituitary adenomas: pathological and clinical implications. Endocr
Relat Cancer 16:1029-1043.

24. Fassnacht M, Capeller B, Arlt W, Steck T, Allolio B (2001) Octreotide LAR treatment throughout pregnancy in an acromegalic woman. Clin Endocrinol (Oxf) 55:411-415.

25. Takano T, Saito J, Soyama A, Ito H, Iizuka T, Yoshida T, Nishikawa T (2006) Normal delivery following an uneventful pregnancy in a Japanese acromegalic patient after discontinuation of octreotide long acting release formulation at an early phase of pregnancy. Endocr $J$ 53:209-212.

26. Maffei P, Tamagno G, Nardelli GB, Videau C, Menegazzo C, Milan G, Calcagno A, Martini C, Vettor R, Epelbaum J, Sicolo N (2010) Effects of octreotide exposure during pregnancy in acromegaly. Clin Endocrinol (Oxf) 72:668-677. 